\title{
A THEORY OF STIEFEL HARMONICS
}

\author{
BY \\ STEPHEN S. GELBART
}

\begin{abstract}
An explicit theory of special functions is developed for the homogeneous space $S O(n) / S O(n-m)$ generalizing the classical theory of spherical harmonics. This theory is applied to describe the decomposition of the Fourier operator on $n \times m$ matrix space in terms of operator valued Bessel functions of matrix argument. Underlying these results is a hitherto unnoticed relation between certain irreducible representations of $S O(n)$ and the polynomial representations of $G L(m, C)$.
\end{abstract}

1. Introduction and notation. The purpose of this paper is to describe an explicit theory of spherical harmonics in the setting of Stiefel manifolds and matrix space. Most of our results have been announced previously in [4].

We let $M_{n, m}$ denote $n \times m$ real matrix space, $S^{n, m}$ the Stiefel manifold of matrices $V \in M_{n, m}$ such that ${ }^{t} V V=I_{m}$, and $P_{m}$ the cone of positive definite $m \times m$ symmetric matrices. Under the action of $S O(n)$ given by left matrix multiplication we identify $S^{n, m}$ with the homogeneous space $S O(n) / S O(n-m)$ provided $n>m$. We denote by $L$ the restriction of the left regular representation of $S O(n)$ to $L^{2}\left(S^{n, m}\right)$ and refer to irreducible representations of $S O(n)$ which occur in $L$ as class $m$ representations. If $f$ is a function on $S^{n, m}$ whose translates under $S O(n)$ span a finite dimensional space equivalent to a multiple of the representation $\omega$ we say that $f$ transforms according to $\omega$. Thus we write

$$
L^{2}\left(S^{n, m}\right)=\sum_{\omega \text { class } m} \bigoplus H_{\omega}^{n, m}
$$

where $H_{\omega}^{n, m}$ consists of functions transforming according to $\omega$. By analogy with the case $m=1$ we call functions in $H_{\omega}^{n, m}$ surface Stiefel harmonics of order $\omega$.

The classical theorems we shall extend are the following:

Theorem 1 (Cartan [3]). Suppose $p$ is a function on the $(n-1)$-sphere $S^{n-1}$ which transforms under $S O(n)$ according to an irreducible representation of highest weight $(k, 0, \ldots, 0)$. Then $p$ extends to a harmonic polynomial on $\mathbf{R}^{n}$ satisfying the homogeneity condition

$$
p(r X)=r^{k} p(X) \text { for } r>0, X \in \mathbf{R}^{n} .
$$

Theorem 1 asserts that a class 1 representation of $S O(n)$ is realizable on a space

Received by the editors September 5, 1972 and, in revised form, March 12, 1973.

AMS (MOS) subject classifications (1970). Primary 43A85, 33A75; Secondary 22E45.

Key words and phrases. Generalized spherical harmonics, Stiefel manifold, representations of $S O(n)$, holomorphic representations of $G L(m, C)$, Fourier transforms on matrix space, generalized Hankel transforms, Bessel functions of matrix argument.

Copyright $\odot 1974$, American Mathematical Society 
of harmonic homogeneous polynomials on $\mathbf{R}^{n}$ (solid spherical harmonics) and that the homogeneity of these polynomials is determined by the highest weight vector of the representation.

Theorem 2 (Bochner [1]). Suppose $f$ is a radial function on $\mathbf{R}^{n}, p$ is as in Theorem 1 , and $F=f p$ is square integrable. Then the Fourier transform of $F$ is $g p$ where $g$ is the Hankel transform of $f$ of order $k+(n-2) / 2$.

Theorem 2 explicitly describes the restriction of the Fourier operator to subspaces of $L^{2}\left(\mathbf{R}^{n}\right)$ transforming according to class 1 representations of $S O(n)$. Both theorems are basic to the classical theory of harmonics and their importance in analysis is well known.

Our motivation for generalizing Theorems 1 and 2 is two-fold. On the one hand it is of intrinsic interest to do explicit harmonic analysis on homogeneous spaces that are not symmetric spaces (e.g., $S^{n, m}$ for $m>1$ ). On the other hand our work has an immediate application to the representation theory of certain noncompact groups. Indeed the so-called "Weil representation" [14] of the real symplectic group $S p(m, \mathbf{R})$ on $L^{2}\left(M_{2 m, m}\right)$ is known to be highly reducible and the theory of harmonics we are about to describe makes it possible to decompose this representation and prove that all the holomorphic discrete series representations of $S p(m, \mathbf{R})$ are contained in it. This work appears in [5].

The contents of this paper may now be summarized as follows. In $\$ 2$ we prove that every surface Stiefel harmonic of order $\omega$ is the restriction to $S^{n, m}$ of a harmonic homogeneous polynomial on $\boldsymbol{M}_{n, m}$. Actually we prove a stronger result. Our solid Stiefel harmonics will be annihilated by all (rather than just one) of the $S O(n)$-invariant differential operators on $M_{n, m}$ and their homogeneity will be described by a simple but hitherto unnoticed relation between class $m$ representations of $S O(n)$ and certain finite dimensional irreducible representations of $G L(m, C)$. In particular we shall prove that the class $m$ representations of $S O(n)$ are realizable on spaces of vector-valued harmonic polynomials on $M_{n, m}$ which transform under $M_{m, m}$ according to appropriate rational representations of $G L(m, C)$. If one interprets the homogeneity condition (1.2) as asserting that $p(X)$ transforms under $M_{1,1}$ according to the representation $z \rightarrow z^{k}$ then this result seems natural. To establish it we shall apply the Frobenius-Young-Schur-Weyl theory of symmetry classes in tensor space.

In $\$ 3$ we shall examine in detail the invariant subspaces of the Fourier transform on $M_{n, m}$. The restriction of the Fourier operator to these subspaces will be described by a generalized Hankel transform on $P_{m}$ whose kernel is defined in terms of a certain integral over $S O(n)$. This kernel is a matrix-valued generalization of the Bessel function. By representing it as an inverse Laplace transform we shall establish (in \$4) generalizations of the classical Bochner relations linking the components of the decomposition of $L^{2}\left(\mathbf{R}^{n}\right)$ for various $n$.

Some examples of generalized spherical harmonics have appeared before in the literature. In [7] Herz introduced a class of homogeneous harmonic polynomials 
on $M_{n, m}$ and (by analytic methods) proved they behave like Stiefel hamonics with respect to the Fourier transform. We shall see that Herz's polynomials correspond to class $m$ representations of $S O(n)$ which occur exactly once in $L^{2}\left(S^{n, m}\right)$. Thus we give a group theoretic explanation of their special behavior with respect to the Fourier transform.

In [9] Levine investigated the Fourier expansion of functions in $L^{2}\left(S^{n, 2}\right)$ and proved by methods of algebraic geometry that harmonics of order $\omega$ on $S^{n, 2}$ extend to harmonic homogeneous polynomials on $M_{n, 2}$. If the highest weight vector of $\omega$ is $\left(m_{1}, m_{2}, 0, \ldots, 0\right)$ then the homogeneity of these polynomials is $m_{1}+\left|m_{2}\right|$. Our Theorem 2.11 contains a group theoretic extension of this result for arbitrary $m$ provided $n \geq 2 m$. Subsequently R. Strichartz has obtained similar results for $2 m>n$ (see [13]).

An important abstract theory of harmonic polynomials on $G$-spaces was developed several years ago by Helgason [6] and Kostant [8]. From Helgason's results it follows that every surface Stiefel harmonic of order $\omega$ is the restriction to $S^{n, m}$ of a harmonic homogeneous polynomial on $M_{n, m}$. However, Helgason neither constructs these polynomials nor describes their homogeneity in terms of $\omega$. It is precisely these refinements of the abstract theory which constitute the contribution of our paper and make possible the application to representation theory pursued in [5].

Once again it is a pleasure for the author to thank Professor E. M. Stein for several valuable conversations and suggestions related to the subject matter of this paper.

2. Harmonic analysis of functions in $L^{2}(S O(n) / S O(n-m))$. In this section we shall describe explicitly the class $m$ representations of $S O(n)$. In particular we shall characterize them in terms of their highest weight vectors and then prove they are realizable on spaces of special harmonic polynomials on $M_{n, m}$ whose restrictions to $S^{n, m}$ yield surface Stiefel harmonics. We shall call these special polynomials solid Stiefel harmonics on $\boldsymbol{M}_{n, \boldsymbol{m}}$.

2.1. Preliminary discussion of class $m$ representations. We start by reviewing some facts from the representation theory of $S O(n)$. If $k=[n / 2]$ then the irreducible representations of $S O(n)$ are in one-to-one correspondence with socalled dominant weight vectors $\omega=\left(m_{1}, \ldots, m_{k}\right)$. These dominant weights are $k$ tuples of integers satisfying

$$
m_{1} \geq m_{2} \geq \cdots \geq m_{k} \geq 0 \text { when } n \text { is odd, }
$$

and

$$
m_{1} \geq m_{2} \geq \cdots \geq m_{k-1} \geq\left|m_{k}\right| \text { when } n \text { is even. }
$$

Without fear of confusion we shall denote the irreducible representation of $S O(n)$ corresponding to the weight $\omega$ by $\omega$ itself. The highest weight vector of this representation is again the $k$-tuple $\omega$. 
Throughout the remainder of $\$ 2$ we shall assume

$$
n>2 m \text {. }
$$

Actually strict inequality in (2.3) is not necessary. Our results do hold for $n=2 m$ (after minor modifications which we include in the form of parenthetical remarks) and it is precisely this case which is crucial to the applications found in [5]. We do not know how to further relax (2.3) so as to include the case of square matrix space (cf. Stein [12, Problem 7]).

Proposition 2.1. If $\omega$ is an irreducible representation of $S O(n)$ with highest weight $\left(m_{1}, \ldots, m_{k}\right)$ then $\omega$ is class $m$ if and only if $m_{j}=0$ for $j>m$. Thus all class $m$ weight vectors are of the form $\left(m_{1}, \ldots, m_{m}, 0, \ldots, 0\right)$.

Proof. Combine an $m$-fold application of the branching theorem for $S O(n)[1$, pp. 251-255] with the Frobenius reciprocity theorem for compact groups.

We now recall how any representation of $S O(n)$ may be realized on a tensor space of appropriate symmetry type. For details and proofs see Weyl [15].

Suppose $\omega=\left(m_{1}, \ldots, m_{k}\right)$ with $m_{k} \geq 0$, let $\nu=m_{1}+\cdots+m_{k}$, and set $P_{v}=Q^{\prime} C^{n}$. The Young diagram corresponding to $\left(m_{1}, \ldots, m_{k}\right)$ is

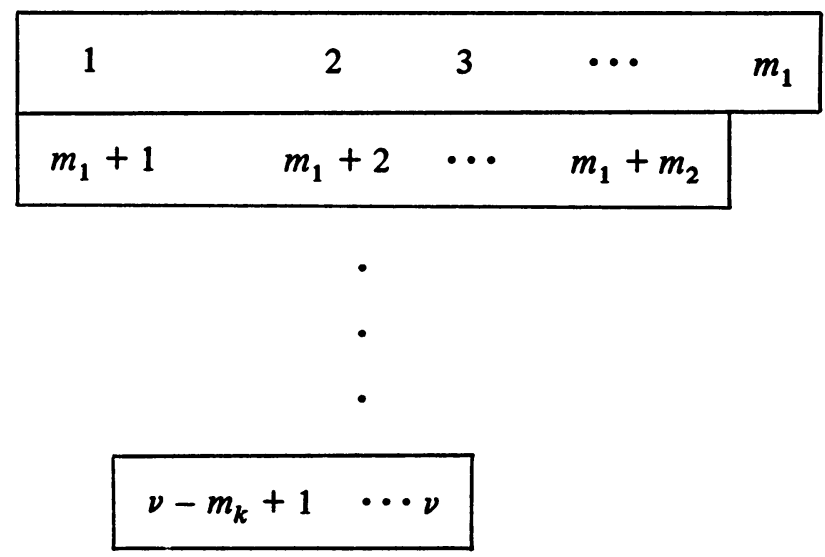

and the symmetrizer corresponding to this diagram is

$$
\varepsilon(\omega)=\sum_{p \in R(\omega) ; q \in C(\omega)} \operatorname{sgn}(q) q p
$$

where $R(\omega)$ (resp. $C(\omega)$ ) denotes the set of all permutations of $1, \ldots, \nu$ preserving the rows (resp. columns) of the diagram. Since the symmetric group $S$, acts on $\otimes^{\prime} C^{n}$ by permutation of the indices, $\varepsilon(\omega)$ maps $P_{r}$ into itself. On the other hand $S O(n)$ (which acts on $P$, via the $\nu$ th Kronecker product of its standard representation) leaves invariant the subspace $\varepsilon(\omega) P_{v}$. The elements of this subspace are called tensors of symmetry class $\omega$. Since $\varepsilon(\omega) P_{v}$ is still not $S O(n)$-irreducible we must further restrict attention to the subspace $P_{v}^{0}$ of $P_{v}$ consisting of traceless 
tensors (tensors in $P$, whose image under any contraction map is zero). Then the representation of $S O(n)$ provided by $\varepsilon(\omega) P_{v}^{0}$ is a multiple of the irreducible representation of highest weight $\omega=\left(m_{1}, \ldots, m_{k}\right)$. (In case $n=2 k$ and $m_{k} \neq 0$, two distinct representations of $S O(n)$ arise in $\varepsilon(\omega) P_{v}^{0}$, one of highest weight $\left(m_{1}, \ldots, m_{k}\right)$, the other of highest weight $\left(m_{1}, \ldots,-m_{k}\right)$, and both occur an equal number of times. Thus the assumption $m_{k} \geq 0$ is not necessary: the irreducible representation of $S O(n)$ indexed by $\left(m_{1}, \ldots, m_{k}\right)$ is realized in a space of traceless tensor of symmetry type $\left(m_{1}, \ldots,\left|m_{k}\right|\right)$ no matter what the sign of $m_{k}$.)

Our goal is to realize the class $m$ representations of $S O(n)$ not on spaces of tensors but on spaces of harmonic homogeneous polynomials on $M_{n, m}$. To do this we must introduce certain representations of $G L(m, C)$ and establish a relation between these representations and the class $m$ representations of $S O(n)$.

We fix, once and for all, an $m$-tuple of integers $\omega=\left(m_{1}, \ldots, m_{m}\right)$ with $m_{1} \geq m_{2} \geq \cdots \geq m_{m} \geq 0$ (respectively $m_{1} \geq m_{2} \geq \cdots \geq\left|m_{m}\right|$ for $n=2 m$ ). By $\rho_{\omega}$ we denote the unique irreducible integral representation of $G L(m, C)$ whose restriction to the unitary group $U(m)$ has highest weight $\left(m_{1}, \ldots, m_{m}\right)$ (resp. $\left(m_{1}, \ldots,\left|m_{m}\right|\right)$ for $\left.n=2 m\right)$. We also consider simultaneously the class $m$ representation of $S O(n)$ of highest weight $\left(m_{1}, \ldots, m_{m}, 0, \ldots, 0\right)$. This representation is well defined by virtue of (2.5). We denote it again by the symbol $\omega$.

The following result seems to be an act of providence.

Proposition 2.2. The class $m$ representation $\omega$ of $S O(n)$ occurs in $L^{2}\left(S^{n, m}\right)$ with multiplicity equal to the dimension of $\rho_{\omega}$.

Proof. The branching theorem for $G L(m, C)$ asserts that if $\rho_{\omega}$ is used as a representation of the subgroup of $G L(m, \mathrm{C})$ isomorphic to $G L(m-1, \mathrm{C})$ then its decomposition contains exactly once every representation $\rho_{\omega}^{\prime}$ of $G L(m-1, \mathrm{C})$ whose indices satisfy $m_{1} \geq m_{1}^{\prime} \geq \cdots \geq m_{m-1}^{\prime} \geq\left|m_{m}\right|$ (cf. [2, p. 161]). On the other hand the branching theorem for $S O(n)$ combined with the Frobenius reciprocity theorem implies that the multiplicity of $\omega$ in $L^{2}\left(S^{n, m}\right)$ equals the multiplicity of the identity representation in the restriction of $\rho_{\omega}$ to the trivial subgroup. Since this multiplicity is simply the dimension of $\rho_{\omega}$ the proof is complete.

Corollary. If $r_{\omega}$ denotes the multiplicity of the class $m$ representation $\omega$ in $L$ (hence also the dimension of $\rho_{\omega}$ ) then

$$
r_{\omega}=\prod_{1 \leq i<j \leq m}\left(m_{i}-m_{j}+j-i\right) / \prod_{1 \leq i<j \leq m}(j-i) .
$$

Proof. The corollary follows from a known dimension formula [2, p. 187] for the integral representations of $G L(m, C)$. (The formula remains valid for $n=2 m$ once $m_{j}$ is replaced by $\left|m_{j}\right|$.)

Remarks. (1) In theory Proposition 2.2 follows directly from an $m$-fold 
application of the branching theorem for $S O(n)$ alone but the computations required are unmanageable.

(2) We believe that Proposition 2.2 is a special case of some general reciprocity phenomenon but we have not been able to make this precise. Because the proposition is crucial to all that follows such an abstract formulation would seem desirable.

To explain the connection between the representations $\rho_{\omega}$ and the theory of solid Stiefel harmonics we are about to describe, we need to introduce some more definitions.

Definition 2.4. We call a function on $M_{n, m} S O(n)$-harmonic if it is annihilated by all $S O(n)$-invariant constant coefficient differential operators without constant term. (These operators are to be thought of as "generalized Laplacians"; cf. Helgason [6] and Kostant [8].)

Definition 2.5. If $\rho_{\omega}(A)$ is some matrix realization of $\rho_{\omega}$ we say that a $C^{t^{\prime}-\text { valued }}$ function on $M_{n, m}$ is homogeneous of degree $\rho_{\omega}(A)$ if the function transforms under $M_{m, m}$ according to $\rho_{\omega}(A)$, i.e.

$$
p(X A)=p(X) \rho_{\omega}(A)
$$

for all $A \in M_{m, m}$. (The matrix $\rho_{\omega}(A)$ is defined for all $m \times m$ complex matrices, invertible or not, because $\rho_{\omega}$ is integral, i.e. $\left|m_{m}\right| \geq 0$.)

Our goal is to prove the existence of a matrix representation $\rho_{\omega}(A)$ and a space $P^{\omega}$ of $\mathbf{C}^{r^{\omega}}$-valued $S O(n)$-harmonic polynomials on $M_{n, m}$ such that

(1) each $p \in P^{\omega}$ is homogeneous of degree $\rho_{\omega}(A)$,

(2) as an $S O(n)$-module, $P^{\omega}$ is equivalent to $\omega$.

To establish this result and the concomitant theory of Stiefel harmonics we shall proceed as follows. First we shall construct a convenient matrix representation $\rho_{\omega}(A)$ and prove that its matrix coefficients can be imbedded in tensor space of

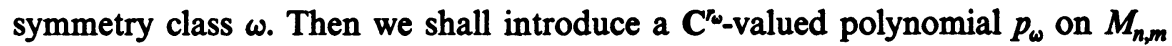
which is $S O(n)$-harmonic and homogeneous of degree $\rho_{\omega}(A)$. The translates of $p_{\omega}(X)$ under $S O(n)$ will span the desired space $P^{\omega}$ and so realize the class $m$ representation $\omega=\left(m_{1}, \ldots, m_{m}, 0, \ldots, 0\right)$. Our solid Stiefel harmonics on $M_{n, m}$ will be defined as coordinate functions of these translates and by homogeneity these harmonics will be determined by their restrictions to $S^{n, m}$.

Our first task then is to construct a convenient matrix representation $\rho_{\omega}(A)$. For this we use the tensor theory developed for this purpose by Frobenius, Schur, Young, and Weyl.

2.2. The construction and description of $\rho_{\omega}(A)$. Recall that $\omega=\left(m_{1}, \ldots, m_{n}\right)$ and set $\nu=m_{1}+\cdots+\left|m_{n}\right|$. If $R_{m^{\prime}}$ denotes the tensor space of $\nu$ th rank belonging to $\mathbf{C}^{m}$ then a standard basis for $R_{m^{\prime}}$ consists of the $m^{y}$ monomials $z_{j_{1}} \cdot z_{j_{2}} \cdots \cdots z_{j_{r}}$ where $1 \leq j_{i} \leq m$. We shall fix this basis once and for all and keep in mind that all that follows depends on this choice of basis.

We consider the $\nu$ th Kronecker product of the standard representation of 
$G L(m, C)$ and denote its matrix (with respect to the above basis) by $T_{p}(A)$. This representation acts on $R_{m}$, but is far from irreducible. Indeed if $\varepsilon(\omega)$ denotes the Young symmetrizer corresponding to $\left(m_{1}, \ldots,\left|m_{n}\right|\right)$ then the subspace $\varepsilon(\omega) R_{m}$ is $G L(m, C)$-invariant and the restriction of $T_{p}(A)$ to $\varepsilon(\omega) R_{m}$, realizes the representation $\rho_{\omega}$. The matrix coefficients of $T_{\nu}(A)$ are obviously monomials of degree $\nu$ in the entries of $A$ but this is not the matrix we are after. We want the $r_{\omega} \times r_{\omega}$ matrix which describes the transformation $T_{p}(A)$ in $\varepsilon(\omega) R_{m}$. Thus we need to construct a basis for $\varepsilon(\omega) R_{m^{\prime}}$ and describe the matrix of $\rho_{\omega}(A)$ with respect to this basis.

Suppose we allow the symmetrizer $\varepsilon(\omega)=\varepsilon\left(m_{1}, \ldots,\left|m_{m}\right|\right)$ to act on the standard basis in $R_{m^{\text {r }}}$. The result is a set of tensors in $\varepsilon(\omega) R_{m^{\prime}}$ which spans $\varepsilon(\omega) R_{m^{\text {r }}}$. From among these tensors (some of which may be zero) we pick $\boldsymbol{r}_{\omega}$ linearly independent ones $t_{1}, \ldots, t_{r_{0}}$ and let $\rho_{\omega}(A)$ denote the matrix of $\rho_{\omega}$ with respect to this basis. We claim that all the entries of $\rho_{\omega}(A)$ belong to the same symmetry class, namely $\omega$, when viewed as tensors in $\otimes^{\prime} \mathrm{C}^{n}$. To see this we make some observations:

(1) If $A=\left(a_{i j}\right)$ is an arbitrary element of $G L(m, C)$ then the action of $A$ on the indeterminants $z_{1}, \ldots, z_{n}$ is given by the formal matrix multiplication

$$
\left(z_{1} \cdots z_{n}\right)\left(\begin{array}{ccc}
a_{11} & \cdots & a_{1 m} \\
\vdots & & \vdots \\
a_{m 1} & \cdots & a_{m m}
\end{array}\right) .
$$

As remarked before the matrix of $T_{p}(A)$ with respect to the basis $\left\{z_{j_{1}} z_{j_{2}} \cdots z_{j_{2}}\right\}_{\left(j_{1}, \ldots, j_{j}\right)}$ consists of monomials of total degree $\nu$ in the $a_{i j}$ (indeed all monomials of this degree which one can construct from the $a_{i j}$ occur). The entries of a fixed column of $T_{v}(A)$ are of the same homogeneity degree $l_{j}$ with respect to the $j$ th column variables $a_{1 j}, \ldots, a_{m j}$ of $A$ and the $l_{j}$ are such that $\sum_{i=1}^{m} l_{j}=\nu$. In fact if $z_{j}$ occurs $l_{j}$ times in $z_{j_{1}} z_{j_{2}} \cdots z_{j_{j}}$, then the entries of $T_{p}(A)$ in the column corresponding to this basis element are homogeneous of order $l_{j}$ in the $j$ th column variables $a_{1 j}, \ldots, a_{m j}$. We say then that this column is of homogeneity type $\left(l_{1}, \ldots, l_{m}\right)$.

(2) Suppose a fixed column of $T_{v}(A)$ is described as above by the homogeneity system $\left(l_{1}, \ldots, l_{m}\right)$. Then each entry of this column may be viewed as an element of the tensor space $\otimes^{\prime} \mathbf{C}^{n}=\left(\otimes^{\prime /} \mathbf{C}^{n}\right) \otimes \cdots \otimes\left(\otimes^{\ln } \mathbf{C}^{n}\right)$ with components $F\left(f_{1}, \ldots, f_{n}\right)$ which are symmetric in $\left\{f_{1}, \ldots, f_{l_{1}}\right\},\left\{f_{l_{1}+1}, \ldots, f_{l_{1}+l_{2}}\right\}, \ldots$, $\left\{f_{v-l_{m-1}+1}, \ldots, f_{v}\right\}$ (cf. Weyl $[15$, p. 96$]$ for terminology).

(3) Each element of the basis $\left\{t_{1}, \ldots, t_{r_{0}}\right\}$ described earlier is the image under $\varepsilon(\omega)$ of some standard basis vector $z_{j_{1}} z_{j_{2}} \cdots z_{j}$ in $R_{m}$. Thus each $t_{i}$ is a linear combination of certain $z_{j_{1}} z_{j_{2}} \cdots z_{j_{j}}$, each of homogeneity type $\left(l_{1}, \ldots, l_{m}\right)$.

Now we can describe the matrix $\rho_{\omega}(A)$ as follows. If $t_{i}$ is a linear combination of certain basis elements $z_{j_{1}} z_{j_{2}} \cdots z_{j_{0}}$ then the nonzero components of the corresponding linear combination of columns of $T_{\nu}(A)$ exhaust the matrix coefficients of the ith column of $\rho_{\omega}(A)$. By observations (1) and (2) these are 
precisely the $\nu$ th rank tensors obtained by applying $\varepsilon(\omega)$ to entries of columns of $T_{v}(A)$ corresponding to this linear combination.

At this stage an example would be illuminating. Fix $m=2, v=3$, and write

$$
A=\left(\begin{array}{ll}
a & b \\
c & d
\end{array}\right)
$$

The standard basis for $R_{2} 3$ is $\left\{z_{1}^{3}, z_{1}^{2} z_{2}, z_{1} z_{2} z_{1}, z_{1} z_{2}^{2}, z_{2} z_{1}^{2}, z_{2} z_{1} z_{2}, z_{2}^{2} z_{1}, z_{2}^{3}\right\}$ and the matrix of $T_{\nu}(A)$ with respect to this basis is

$\left[\begin{array}{llllllll}a^{3} & a^{2} b & a^{2} b & a b^{2} & a^{2} b & a b^{2} & a b^{2} & b^{3} \\ a^{2} c & a^{2} d & a b c & a b d & a b c & a b d & b^{2} c & b^{2} d \\ a^{2} c & a b c & a^{2} d & a b d & a b c & b^{2} c & a b d & b^{2} d \\ a c^{2} & a c d & a c d & a d^{2} & b c^{2} & b c d & b c d & b d^{2} \\ a^{2} c & a b c & a b c & b^{2} c & a^{2} d & a b d & a b d & b^{2} d \\ a c^{2} & a c d & b c^{2} & b c d & a c d & a d^{2} & b c d & b d^{2} \\ a c^{2} & b c^{2} & a c d & b c d & a c d & b c d & a d^{2} & b d^{2} \\ c^{3} & c^{2} d & c^{2} d & c d^{2} & c^{2} d & c d^{2} & c d^{2} & d^{3}\end{array}\right]$.

Now fix $\omega=(2,1)$ and consider the diagram

\begin{tabular}{|l|l|}
\hline 1 & 2 \\
\hline 3 & \\
\hline
\end{tabular}

The space $\varepsilon(\omega) R_{2} 3$ is 2 -dimensional and spanned by the linearly independent vectors $t_{1}=z_{1}^{2} z_{2}-z_{2} z_{1}^{2}$ and $t_{2}=z_{1} z_{2}^{2}-z_{2}^{2} z_{1}$. The resulting matrix of $\rho_{\omega}$ with respect to $\left\{t_{1}, t_{2}\right\}$ is

$$
\rho_{\omega}(A)=\left(\begin{array}{ll}
a^{2} d-a b c & a b d-b^{2} c \\
a c d-b c^{2} & a d^{2}-b c d
\end{array}\right)
$$

obtained by subtracting first the second and fifth columns of $T_{v}(A)$, then the fourth and seventh. Observe that as tensors in $\otimes^{3} C^{2}$ (or $\otimes^{3} C^{n}$ ) the entries of $\rho_{\omega}(A)$ belong to the symmetry class $\omega=(2,1)$. Also note that $\rho_{\omega}(A)$ is hermitian whenever $A$ is. The property of being hermitian is defined in terms of the inner product on $\otimes^{\prime} C^{n}$ adapted to the representation $\rho_{\omega}$ in the sense of Godement (Séminaire H. Cartan, 10e année: 1957/58, p. 5-08). This is the (essentially unique) inner product with respect to which the restriction of $\rho_{\omega}$ to $U(m)$ is unitary.

We summarize the general phenomenon in

Theorem 2.6. The coefficients of the matrix $\rho_{\omega}(A)$ just constructed belong to the symmetry class $\omega$ when viewed as tensors in $\otimes^{\prime} C^{n}$. 
2.3. Explicit description of class $m$ representations of $S O(n)$. As promised, we shall use Theorem 2.6 to establish the following proposition.

Proposition 2.7. There exists a polynomial $p_{\omega}: M_{n, m} \rightarrow C^{r_{\omega}}$ such that:

(1) $p_{\omega}(X)$ is $S O(n)$-harmonic,

(2) $p_{\omega}(X)$ is homogeneous of degree $\rho_{\omega}(A)$, and

(3) the components of $p_{\omega}(X)$ lie in $\varepsilon(\omega) P_{\nu}^{0}$ when viewed as tensors.

To prove proposition 2.7 we need a lemma which is interesting in its own right.

Lemma 2.8. Suppose $\rho_{\omega}(A)$ is a matrix representation of $G L(m, C)$ and $p: M_{n, m}$ $\rightarrow \mathbf{C}^{r_{\omega}}$ is a polynomial homogeneous of degree $\rho_{\omega}(A)$. Then if $p(X)$ is harmonic in the ordinary sense it is also $S O(n)$-harmonic.

Proof. The ring of all $S O(n)$-invariant constant coefficient differential operators on $M_{n, m}$ without constant term is generated by the operators

$$
\Delta_{i l}=\sum_{k=1}^{n} \frac{\partial}{\partial x_{k i}} \frac{\partial}{\partial x_{k l}}, \quad 1 \leq i, l \leq m .
$$

To say that $p$ is harmonic is to assume $\sum_{i=1}^{n} \Delta_{i i} p \equiv 0$. To prove that $p$ is $S O(n)$ harmonic it suffices to show $\Delta_{i l} p \equiv 0$ for all $i, l$.

Write $A=\left(a_{i j}\right)$ for $A \in M_{m, m}$. Set $g(X)=p_{\omega}(X A)=p_{\omega}(X) \rho_{\omega}(A)$. By the chain rule, $g^{\prime}(X)=p^{\prime}(X A) A^{\prime}(X)$ where $A^{\prime}(X)$ is the Jacobian of the map $A: M_{n, m} \rightarrow M_{n, m}$ which takes $X=\left(x_{i j}\right)$ into $X^{\prime}=\left(x_{i j}^{\prime}\right)=\sum_{p-1}^{m} x_{i p} a_{p j}$, and $p^{\prime}(X A)$ equals

$$
\left(\frac{\partial p}{\partial x_{11}}(X A) \cdots \frac{\partial p}{\partial x_{n 1}}(X A) \frac{\partial p}{\partial x_{12}}(X A) \cdots \frac{\partial p}{\partial x_{n m}}(X A)\right)
$$

Thus direct computation of $A^{\prime}(X)$ yields

$$
\frac{\partial g}{\partial x_{k l}}(X)=\sum_{p=1}^{m} a_{l p} \frac{\partial p}{\partial x_{k p}}(X A) .
$$

If we now fix

$$
A^{i}=\left(\begin{array}{ccccccc}
1 & & & & & & \\
& \ddots & & & & 0 & \\
& & 1 & & & & \\
& & & a_{i i} & & & \\
& & & & 1 & & \\
0 & & & & & \ddots & \\
& & & & & & 1
\end{array}\right)
$$

with $a_{i i}$ arbitrary but not equal to zero then (2.6) becomes $\left(\partial g / \partial x_{k l}\right)(X)$ $=a_{l l}^{*}\left(\partial p / \partial x_{k l}\right)\left(X A^{i}\right)$ where $a_{l l}^{*}=1$ for $l \neq i$ and $a_{l l}^{*}=a_{i i}$ for $l=i$. Thus 


$$
\left(\partial^{2} g / \partial x_{k l}^{2}\right)(X)=\left(a_{l l}^{*}\right)^{2}\left(\partial^{2} p / \partial x_{k l}\right)\left(X A^{i}\right) .
$$

Applying $\Delta=\sum_{i=1}^{m} \Delta_{i i}$ to both sides of the equation

$$
g(X)=p_{\omega}(X) \rho_{\omega}(A)
$$

we obtain

$$
\sum_{l \neq i} \sum_{k=1}^{n} \frac{\partial^{2} p}{\partial x_{k l}}(X A)+a_{i i}^{2} \sum_{k=1}^{n} \frac{\partial^{2} p}{\partial x_{k l}^{2}}(X A)=0
$$

since $p_{\omega}(X)$ is harmonic. But $X A$ exhausts all of $M_{n, m}$ as $X$ does since $\operatorname{det}(A) \neq 0$. Thus (2.8) is valid with $X A$ replaced by $X$ and we may conclude (from the arbitrariness of $a_{i i}$ ) that

$$
0 \equiv \sum_{l \neq i} \sum_{k=1}^{n} \frac{\partial^{2} p}{\partial x_{k l}}(X A)=\sum_{k=1}^{n} \frac{\partial^{2} p}{\partial x_{k l}^{2}}(X)=\left(\Delta_{l l} p\right)(X) .
$$

It remains to prove that $\Delta_{i l} p \equiv 0$ for $i \neq l$. For this we pick

$$
A=A^{1 j}=\left(\begin{array}{cccccc}
1 & 0 & \cdots & 1 & \cdots & 0 \\
& \ddots & & & \\
\vdots & & & 1 & & 0 \\
& & & \ddots & \\
0 & & & \cdots & & 1
\end{array}\right)
$$

with the extra 1 in the $j$ th column, $j \neq 1$. Since $\operatorname{det}\left(A^{1 j}\right) \neq 0$, an argument similar to the one just used shows that $\Delta_{\mathrm{l} j} p \equiv 0$. Now fix

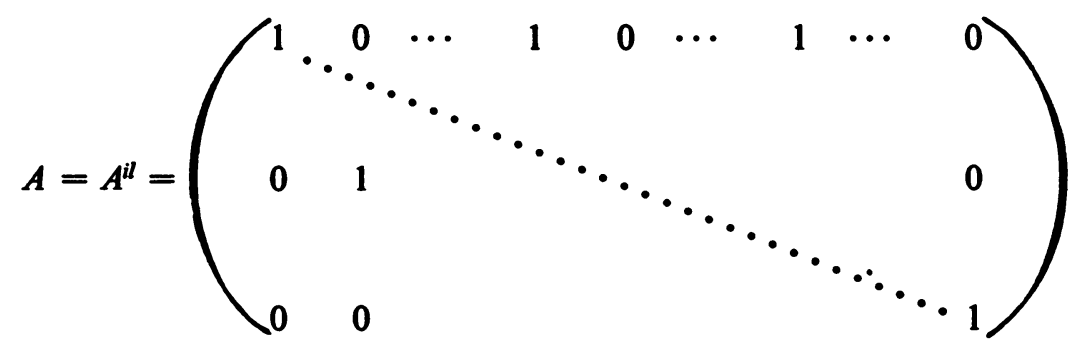

(an extra 1 in both the $i$ th and $l$ th columns). By a similar but more complicated argument the desired conclusion $\Delta_{i l} p \equiv 0$ follows from the already established fact that $\Delta_{i i} p \equiv 0 \equiv \Delta_{1 j} p$.

Proof of Proposition 2.7. Define a map Z: $M_{n, m} \rightarrow M(m, \mathrm{C})$ by

$$
\begin{aligned}
Z(X) & =Z\left(\begin{array}{ccc}
x_{11} & \cdots & x_{1 m} \\
\cdot & & \cdot \\
\cdot & & \cdot \\
\cdot & \ldots & \cdot \\
x_{n 1} & \cdots & x_{n m}
\end{array}\right)=\left(z_{k l}\right) \cdot \\
& =\left(x_{2 k-1, l}+(-1)^{1 / 2} x_{2 k, l}\right), \quad 1 \leq k, l \leq m .
\end{aligned}
$$


(This makes sense since $n \geq 2 m$.) Now let $p_{\omega}(X)$ denote the first row of $\rho_{\omega}(Z(X))$.

We claim that $p_{\omega}(X)$ satisfies properties (1)-(3) of Proposition 2.7. Indeed property (2) follows from the fact that $Z(X A)=Z(X) A$ and $\rho_{\omega}$ is a representation.

Each coordinate function of $p_{\omega}(X)$ is harmonic on $M_{n, m}$ since each matrix coefficient of $\rho_{\omega}(Z)$ is polynomial and hence holomorphic in $z_{k l}$. Property (1) of the proposition thus follows from Lemma 2.8 .

Finally observe that $S O(n)$-harmonic polynomials on $M_{n, m}$ may be viewed as traceless tensors since $i-l$ contractions correspond to operators $\Delta_{i l}$. Since Theorem 2.6 implies the coordinate functions of $p_{\omega}(X)$ must belong to the symmetry class $\omega=\left(m_{1}, \ldots,\left|m_{m}\right|, 0, \ldots, 0\right)$ these functions must lie in $\varepsilon(\omega) P_{0}^{0}$ when viewed as tensors in $\otimes^{\nu} \mathbf{C}^{n}$. This completes the proof.

Definition 2.9. Let $P^{\omega}$ denote the linear space of translates under $S O(n)$ of the special polynomial $p_{\omega}(X)$ of Proposition 2.7. The space $\mathcal{T}_{\omega}^{n, m}$ of solid Stiefel harmonics of order $\omega$ on $M_{n, m}$ is defined to be the direct sum of the space ${ }^{3} \omega_{\omega}^{j}$ of $j$ th coordinate entries of functions in $P^{\omega}$.

Remarks. (1) If $m=1$ and $\omega=k$ then $P^{\omega}$ is the classical space of solid spherical harmonics of order $k$ on $\mathbf{R}^{n}$.

(2) It follows from the construction of $\rho_{\omega}(A)$ that a translate of $p_{\omega}(X)$ vanishes identically if and only if each of its coordinate functions does. Thus there is an obvious isomorphism between $P^{\omega}$ and each $\mathcal{S}_{\omega^{j}}^{j}$.

(3) The components of any row of the matrix $\rho_{\omega}(A)$ (as opposed to $T_{\nu}(A)$ ) are distinct (and more often than not of distinct homogeneity type; cf. (2.6) and (2.7)). Thus the entries of $p_{\omega}(X)$ are distinct and transform differently under the action of $M_{m, m}$. In particular the spaces $\mathcal{F}_{\omega}^{j}$ just defined are pairwise disjoint.

Theorem 2.10. The space $P^{\omega}$ realizes the irreducible representation $\omega$ of $S O(n)$ of highest weight $\left(m, \ldots,\left|m_{m}\right|, 0, \ldots, 0\right)$.

Proof. Recall that every function in $P^{\omega}$ is homogeneous of degree $\rho_{\omega}(A)$. Since $M_{n, m}=S^{n, m} \times P_{m}$ this implies that every such function is determined by its restriction to $S^{n, m}$. In particular, by Remark 2, the map $\pi$ restricting functions in $\mathcal{F}_{\omega}^{j}$ to $S^{n, m}$ is an isomorphism, and $P^{\omega}$, as an $S O(n)$-module, is equivalent to each $\pi\left(\mathcal{H}_{\omega}^{j}\right)$. Let $H_{\omega}$ denote $\pi\left(\mathcal{F}_{\omega}^{j}\right)$. By Proposition 2.7 every function in $\mathcal{F}_{\omega}^{j}$ may be viewed as an element of $\varepsilon(\omega) P_{r}^{0}$. Thus each of the $r_{\omega}$ copies of $H_{\omega}^{j}$ (distinct by Remark (3)) realizes a multiple of the irreducible representation $\omega$. (When $n=2 m, P^{\omega}$ realizes a multiple of $\left(m_{1}, \ldots,\left|m_{m}\right|\right)$.) But $\omega$ occurs exactly $r_{\omega}$ times in $H_{\omega}^{n, m}$ (Proposition 2.2). Thus $\sum_{j=1}^{r_{\omega}} \bigoplus H_{\omega}^{j}=H_{\omega}^{n, m}$ and $P^{\omega}$ realizes $\omega$ just once.

Theorem 2.11. Any Stiefel harmonic of order $\omega$ on $S^{n, m}$ extends to a solid Stiefel harmonic of the same order.

Proof. It has already been remarked that the homogeneity built into the function $p_{\omega}(X)$ implies that coordinate entries of functions in $P^{\omega}$ are determined 
by their restrictions to $S^{n, m}$. Thus

$$
\pi\left(\sum_{j=1}^{r_{\omega}} \bigoplus \mathcal{H}_{\omega}^{j}\right)=\sum_{j=1}^{r_{\omega}} \bigoplus H_{\omega}^{j}
$$

But from the proof of Theorem 2.10 we know that $\sum_{j=1}^{r_{j}} \bigoplus H_{\omega}^{j}$ exhausts the space $H_{\omega}^{n, m}$ of surface Stiefel harmonics of order $\omega$. This completes the proof of Theorem 2.11.

Remarks. (1) The space $P^{\omega}$ obviously depends on a choice of basis. However the space $\mathcal{K}_{\omega}^{n, m}$ does not-it is independent of the choice of $P^{\omega}$. Therefore it is meaningful to refer to $\mathcal{H}_{\omega}^{n, m}$ as the space of solid Stiefel harmonics of order $\omega$.

(2) Every solid harmonic of order $\omega$ is homogeneous of degree $\nu=m_{1}+\cdots$ $+\left|m_{m}\right|$ in the variables $x_{i, j}$ but two such harmonics need not be of the same homogeneity type. That is, they may be of different homogeneity degrees with respect to the various column variables.

We have now shown that functions in $L^{2}\left(S^{n, m}\right)$ may be expanded in terms of restrictions to $S^{n, m}$ of $S O(n)$-harmonic homogeneous polynomials on $M_{n, m}$. To prepare for a generalization of Bochner's Theorem 2 we shall need to strengthen Theorem 2.11.

For this we fix an inner product on $P^{\omega}$ as follows. If $\left\{z_{1}, \ldots, z_{n}\right\}$ denotes the standard orthonormal basis for $\mathbf{C}^{n}$ then $\left\{z_{j_{1}} z_{j_{2}} \cdots z_{j_{j}}\right\}$ is an orthonormal basis for $Q^{\prime} C^{n}$. The inner product of two functions in $P^{\omega}$ is given by the inner product of their corresponding first coordinates viewed as tensors in $\Theta^{\prime} \mathbf{C}^{n}$. Because $\omega$ is irreducible this is (up to a scalar factor) the only $S O(n)$-invariant inner product on $P^{\omega}$.

Now let $F^{\omega}$ denote the space of $S O(n-m)$-invariant functions in $P^{\omega}$ and set $\overline{1}$ equal to

$$
\left(\begin{array}{c}
I_{m} \\
O_{n-m, m}
\end{array}\right)
$$

Since $P^{\omega}$ (as an $S O(n)$-module) is equivalent to $\omega=\left(m_{1}, \ldots, m_{m}, 0, \ldots, 0\right)$ there are precisely $r_{\omega}$ linearly independent elements in $F^{\omega}$. Suppose $\left\{p_{1}, \ldots, p_{d_{\omega}}\right\}$ is a basis for $P^{\omega}$ with the following properties:

(1) $p_{1}, \ldots, p_{r_{\omega}}$ belong to $F^{\omega}$;

(2) $\quad p_{l}(\overline{1})=(0 \cdots 10 \cdots)$ for $1 \leq l \leq r_{\omega}$; and

(3) $p_{l} \perp p_{j}$ for $l \leq r_{\omega}, j>r_{\omega}$.

The existence of such a basis is assured by the following lemma.

Lemma 2.12. Let $\left\{p_{1}, \ldots, p_{r_{\omega}}\right\}$ (resp. $\left.\left\{p_{r_{\omega}+1}, \ldots, p_{d_{\omega}}\right\}\right)$ denote a basis for $F^{\omega}$ (resp. the orthocomplement of $\left.F^{\omega}\right)$. Then:

(a) $p_{l}(1)=(0 \cdots 0)$ whenever $l>r_{\omega}$; and

(b) the vectors $p_{1}(1), \ldots, p_{r_{\omega}}(1)$ are linearly independent in $\mathbf{C}^{r_{\omega}}$. 
Proof. To prove (a) let $\left(t_{j}^{\omega}(O)\right)$ be the matrix defined by

$$
p_{j}\left(O^{-1} X\right)=\sum_{l=1}^{d_{\omega}} t_{l j}^{\omega}(O) p_{l}(X) .
$$

Since $S O(n-m)$ fixes $F^{\omega}$ pointwise and leaves invariant its orthocomplement the restriction of $\left(t_{i j}^{\omega}(O)\right)$ to $S O(n-m)$ must be of the form

$$
\left(\begin{array}{cc}
I_{r_{\omega}} & 0 \\
0 & t_{l j}^{\omega}(O)
\end{array}\right) \text {. }
$$

Now suppose that for $l>r_{\omega}$ one of the components of $p_{l}(1)$ is not zero (we may suppose it is the first component). According to (2.10), with $O \in S O(n-m)$ and $X=\overline{1}$,

$$
p_{j}(\overline{1})=\sum_{l=1}^{d_{\omega}} t_{j}^{\omega}(O) p_{l}(\overline{1}) .
$$

But if $j>r_{\omega}$ it follows from (2.11) that

$$
\overline{p_{j}}(\overline{1})=\sum_{l=r_{0}+1}^{d_{\omega}} t_{j j}^{\omega}(O) p_{l}(\overline{1}) .
$$

Thus

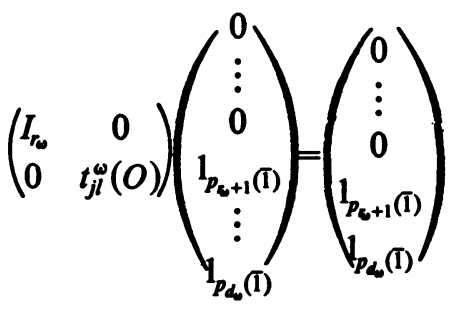

where ${ }^{1} p_{l}(\overline{1})$ denotes the first component of $p_{l}(\overline{1})$. That is, the space of vectors in $C^{d \omega}$ which are fixed by $O \in S O(n-m)$ has dimension at least $\omega_{\omega}+1$. This contradiction establishes part (a) of the lemma.

To prove (b) it suffices to show that $p_{l}(\overline{1})$ cannot possibly equal $(0 \cdots 0)$ if $l \leq r_{\omega}$. By part (a) and (2.10) we have

$$
\begin{aligned}
p_{j}\left(O^{-1} \overline{1}\right) & =\sum_{l=1}^{r_{i}^{\omega}} t_{j j}^{\omega}(O) p_{l}(\overline{1}) \quad \text { or } \\
p_{j}(O \overline{1}) & =\left(\sum_{l=2}^{r_{\omega}} t_{l j}^{\omega}\left(O^{-1}\right)^{1} p_{l}(\overline{1}) \cdots \sum_{l=2}^{n \omega} t_{j j}^{\omega}\left(O^{-1}\right)^{1} p_{1}(\overline{1})\right)
\end{aligned}
$$

assuming $p_{1}(1)=(0 \cdots 0)$.

We claim that the coefficients of $\left(t_{j j}^{\omega}\left(O^{-1}\right)\right)$ are linearly independent and span $H_{\omega}^{n, m}$ as $l$ runs from 1 to ${ }_{\omega}$ and $j$ runs from 1 to $d_{\omega}$. Indeed the identity 


$$
t_{l j}^{\omega}\left(\left(g^{-1} O\right)^{-1}\right)=\sum_{k=1}^{d_{\omega}} t_{k j}(g) t_{l k}\left(O^{-1}\right)
$$

implies that each row space of $\left(t_{j j}^{\omega}\left(O^{-1}\right)\right)$ is $S O(n)$-invariant and that its entries transform according to the representation $\omega$. Similarly the identity

$$
t_{l j}^{\omega}\left((O g)^{-1}\right)=\sum_{k=1}^{d_{\omega}} t_{k k}^{\omega}\left(g^{-1}\right) t_{k j}^{\omega}\left(O^{-1}\right)
$$

implies that $t_{j j}^{\omega}\left(O^{-1}\right)$ is right $S O(n-m)$-invariant and a function on $S^{n, m}$ for $l \leq r_{\omega}$ (for $j \leq r_{\omega}, t_{l j}^{\omega}\left(O^{-1}\right)$ is also left $S O(n-m)$-invariant). Thus our claim is established.

To reach a contradiction we argue as follows. According to an earlier remark the coordinate entries of $p_{j}(O \overline{1})$ must generate all of $H_{\omega}^{n, m}$. But the above claim together with (2.12) indicates that this is impossible. Thus part (b) of the lemma is proved.

The existence of a basis satisfying (2.9) is now assured. One chooses functions $p_{1}(X)$ through $p_{r_{0}}(X)$ in $F^{\omega}$ such that

$$
\left(\begin{array}{c}
p_{1}(\overline{1}) \\
\vdots \\
p_{r_{0}}(\overline{1})
\end{array}\right)=I_{r_{00}}
$$

and extends to a basis $\left\{p_{1}, \ldots, p_{d_{u}}\right\}$ in $P^{\omega}$ with $\left\{p_{r_{u}+1}, \ldots, p_{d_{u}}\right\}$ a basis for the orthocomplement of $F^{\omega}$. Note that the functions $p_{1}, \ldots, p_{r_{0}}$ are uniquely determined by the condition (2.13) and depend ultimately on the choice of matrix representation $\rho_{\omega}(A)$. (As the referee has pointed out, the existence of a basis satisfying (2.9) follows from a more general argument valid for arbitrary compact groups. We have retained our original argument (Lemma 2.12) because it is more in line with the spirit of this paper and because it collects some facts which will be used in the sequel.)

Now let $\omega(O)=\left(t_{i j}^{\omega}(O)\right)$ denote the matrix of $S O(n)$ acting on $P^{\omega}$ with respect to the basis $\left\{p_{1}, \ldots, p_{d_{d}}\right\}$. By $(2.10)$

$$
p_{j}\left(O^{-1} \overline{1}\right)=\left(t_{1 j}^{\omega}(O) t_{2 j}^{\omega}(O) \cdots t_{r_{\omega j}}^{\omega}(O)\right) .
$$

Thus we have:

Theorem 2.13. If $\omega^{*}(O)$ denotes the upper left-hand $r_{\omega} \times r_{\omega}$ submatrix of the matrix representation $\omega(O)$ just described then $\omega^{*}\left(O^{-1}\right)^{t}$ is well defined as a function of $S^{n, m}$

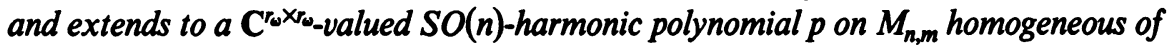
degree $\rho_{\omega}(A)$.

Remark. In general the matrix coefficients of $\omega^{*}\left(O^{-1}\right)^{t}$ may not be orthogonal functions in $L^{2}\left(S^{n, m}\right)$. Indeed it follows from the irreducibility of $\omega$ that the columns of $\omega^{*}\left(O^{-1}\right)^{t}$ are not orthogonal unless the functions $p_{1}, \ldots, p_{r_{0}}$ are. On 
the other hand suppose $r_{\omega}=1$. Then $\omega^{*}\left(O^{-1}\right)^{t}$ and $\rho_{\omega}(A)$ are uniquely determined scalar functions and $\left\{p_{2}, \ldots, p_{d_{\omega}}\right\}$ may be so chosen that $\left(t_{i j}^{\omega}(O)\right)$ is unitary. This basis free situation is worth special attention.

Theorem 2.14. Suppose $\omega=\left(m_{1}, \ldots, m_{m}, 0, \ldots, 0\right)$ is such that $r_{\omega}=1$. Then $m_{1}=m_{2}=\cdots=\left|m_{m}\right|=k$ for some $k \geq 0$ and any harmonic of order $\omega$ on $S^{n, m}$ extends to a so-called $H$-polynomial on $M_{n, m}$ that is, a polynomial $p$ such that

(1) $p$ is harmonic, and

(2) $p$ is determinantally homogeneous of order $k$, i.e. $p(X A)=(\operatorname{det} A)^{k} p(X)$ for all $X \in M_{n, m}$ and $A \in M_{m, m}$. Conversely the restriction of an $H$-polynomial to $S^{n, m}$ is a surface Stiefel harmonic of order $\left(m_{1}, \ldots, m_{m}, 0, \ldots, 0\right)$ with $\left|m_{i}\right|=k$.

Proof. The assertion $m_{1}=\cdots=\left|m_{m}\right|=k$ follows from Corollary 2.3. Since $r_{\omega}=1$ we must have $\rho_{\omega}(A)=(\operatorname{det} A)^{k}$. Thus the first part of Theorem 2.14 is a special case of Theorem 2.13. Note $\omega^{*}\left(O^{-1}\right)^{t}$ generates the space of all harmonics of order $\omega$ on $S^{n, m}$ and is independent of a choice of basis.

For the converse observe that $H$-polynomials (by homogeneity) are determined by their restriction to $S^{n, m}$. Now assume $m=1$. From (1) and (2) it follows that $p$ may be viewed as a traceless tensor in $\otimes^{k} C^{n}$ which transforms under $S O(n)$ according to a single representation $\omega^{\prime}=\left(k^{\prime}, 0, \ldots, 0\right)$. Thus the restriction of $p$ to $S^{n, 1}$ is a harmonic of order $\omega^{\prime}$ and our theory tells us that $p$ must satisfy the homogeneity condition $p(r X)=r^{k^{\prime}} p(X)$ for all $r$. In particular $\omega^{\prime}$ must equal $\omega=(k, 0, \ldots, 0)$. For $m \geq 1$ we reach a similar conclusion by appealing to Lemma 2.8 to show $p$ is traceless tensor in $\otimes^{m k} C^{n}$ and by replacing the condition $p(r X)=r^{k^{\prime}} p(X)$ by a more complicated one.

The $H$-polynomials of Theorem 2.14 were introduced first in [7]. Our theory assigns them a group theoretic significance:

Corollary 2.15. H-polynomials are characterized as extensions to $M_{n, m}$ of Stiefel harmonics of order $\omega$ with $r_{\omega}$ equal to 1.

In case $m=1$ the multiplicity $r_{\omega}$ is always 1 . Thus Corollary 2.15 yields the familiar characterization of solid spherical harmonics of order $k$. At the moment we do not know how to characterize solid harmonics of arbitrary order. One should be able to prove that linear combinations of solid Stiefel harmonics generate all $S O(n)$-harmonic polynomials on $M_{n, m}$ and that all such polynomials are determined by their restriction to $S^{n, m}$. (For $m=1$ this is obvious; for $m=2$ this has been established in [9].) E.M. Stein has suggested that every solid Stiefel harmonic is a linear combination of polynomials of the form $\left(\sum_{j=1}^{m} \sum_{i=1}^{n} a_{i j} x_{i j}\right)^{k}$ where $A=\left(a_{i j}\right)$ satisfies $A A=0$ with $a_{i j} \in \mathbf{C}$. The work of Helgason [6] and Kostant [8] makes this conjecture seem correct.

3. Decomposition of the Fourier transform on matrix space. In this section $M_{n, m}$ denotes $n \times m$ real matrix space with $n \geq m$. We recall that almost every $X \in M_{n, m}$ may be written uniquely in the form $X=V R^{1 / 2}$ with $V \in S^{n, m}$ and 
$R \in P_{m}$. By $d O$ we denote the element of normalized Haar measure on $S O(n)$ and by $d V$ the invariant measure on $S^{n, m}$ normalized so that

$$
\int f(V) d V=\int f(O \overline{1}) d O
$$

for all continuous functions $f$ on $S^{n, m}$. The integration formula accompanying the decomposition $M_{n, m}=S^{n, m} \times P_{m}$ is

$$
\int_{M_{n, m}} F(X) d X=c_{n, m} \int_{R_{m}}\left(\int_{S^{n, m}} F\left(V R^{1 / 2}\right) d V\right)(\operatorname{det} R)^{y} d R
$$

where $\nu=(n-(m+1)) / 2, c_{n, m}$ is a constant depending only on $n$ and $m$, and $d R=\Pi_{i<j} d r_{i j}$ if $R=\left(r_{i j}\right) \in P_{m}$. If ${ }^{*} \mathcal{C}_{\omega}^{n, m}$ denotes the subspace of $L^{2}\left(M_{n, m}\right)$ which transforms under $S O(n)$ according to the irreducible representation $\omega$ then

$$
L^{2}\left(M_{n, m}\right)=\sum_{\omega} \bigoplus * \mathcal{F}_{\omega}^{n, m}
$$

Proposition 3.1. The space ${ }^{*} \mathrm{C}_{\omega}^{n, m}$ is nonempty only if $\omega$ is a class $m$ representation. In this case ${ }^{*} \mathcal{C}_{\omega}^{n, m}$ consists of all functions of the form

$$
F(X)=F\left(V R^{1 / 2}\right)=\sum_{i=1}^{n} \sum_{j=1}^{d} f_{i j}(R) t_{i j}^{\omega}\left(O^{-1}\right)
$$

where $f_{i j} \in L_{n, m}^{2}(\nu)=L^{2}\left(P_{m}, c_{n, m}(\operatorname{det} R)^{y} d R\right)$ and $\left(t_{i j}^{\omega}(O)\right)$ is any matrix representation of $S O(n)$ which is equivalent to $w$ and such that

$$
\left(t_{i j}^{\omega}(O)\right)=\left(\begin{array}{cc}
I_{r_{\omega}} & 0 \\
0 & t_{i j}^{\omega}(O)
\end{array}\right) \text { for all } 0 \in S O(n-m) .
$$

(Note that when $n=m$ condition (3.5) is superfluous and ${ }^{*} \mathcal{J}_{\omega}^{n, m}$ is nonempty for all w.)

Proof. We know that $L^{2}\left(S^{n, m}\right)=\Sigma_{\omega} \oplus H_{\omega}^{n, m}$, the summation extending over all class $m$ representations $\omega$ of $S O(n)$. But the space generated by the matrix entries of the first $r_{\omega}$ rows of $t_{i j}^{\omega}\left(O^{-1}\right)$ exhausts $H_{\omega}^{n, m}$. Thus the proof is complete once we observe that (3.2) implies that $L^{2}\left(M_{n, m}\right)=L^{2}\left(S^{n, m}\right) \otimes L_{n, m}^{2}(v)$.

Remark. If $\omega=(0, \ldots, 0)$ then ${ }^{*} \mathcal{C}_{\omega}^{n, m}$ consists of radial functions on $M_{n, m}$. (A function $R \in L^{2}\left(M_{n, m}\right)$ is radial if and only if $F\left(O^{-1} X\right)=F(X)$ for all $O$ $\in S O(n)$, or equivalently, $F(X)=f^{\prime}(X X)$ for some $f$ on $P_{m}$.)

The Fourier operator $\mathscr{F}$ is defined on smooth compactly supported functions on $M_{n, m}$ by the formula

$$
\mathscr{G} F(Y)=f(Y)=\int_{M_{m, m}} F(X) e^{2 \pi i(X, Y)} d X
$$

where $(X, Y)=\operatorname{trace}\left(X Y^{\imath}\right)$. By Plancherel's theorem $\mathscr{F}$ extends to a unitary 
operator on $L^{2}\left(M_{n, m}\right)$ which is easily seen to commute with rotations. Thus $\mathscr{F}$ preserves the decomposition (3.3).

Theorem 3.2. Suppose $F$ in ${ }^{*} \mathcal{C}_{\omega}^{n, m}$ corresponds to the matrix $\left(f_{i j}\right)$ (as in (3.4)). Let $S_{\omega}^{n, m}(R, P)$ on $P_{m} \times P_{m}$ be defined by

$$
S_{\omega}^{n, m}(R, P)=c_{n, m} \int_{S O(n)} \omega^{*}\left(O^{-1}\right)^{t} \exp \left(2 \pi i \operatorname{trace}\left({ }^{t} \overline{1} O \overline{1} R^{1 / 2} P^{1 / 2}\right)\right) d O
$$

where $\omega^{*}(O)$ is the $r_{\omega} \times r_{\omega}$ upper left-hand submatrix of $\left(t_{i j}^{\omega}(O)\right)$. Then FF again belongs to ${ }^{*} \mathcal{C}_{\omega}^{n, m}$ and corresponds to the matrix $\left(g_{i j}\right)$ where

$$
\left(g_{i j}(P)\right)=\int S_{\omega}^{n, m}(R, P)\left(f_{i j}(R)\right)(\operatorname{det} R)^{\prime} d R .
$$

Proof. Suppose $X=O \overline{1} R^{1 / 2}$ and $Y=K \overline{1} P^{1 / 2}$. By Proposition 3.1 we may assume $F$ is of the form $\sum_{i=1}^{r_{\omega}} \sum_{j=1}^{d_{\omega}} f_{i j}(R) t_{j j}^{\omega}\left(O^{-1}\right)$ where the matrix $\left(t_{i j}^{\omega}(O)\right)$ satisfies (3.5). So by formulas (3.1), (3.2), and (3.6),

$$
\begin{aligned}
F(Y)=c_{n, m} & \sum_{i=1}^{r_{\omega}} \sum_{j=l}^{d_{\omega}} \int_{R_{m}} f_{i j}(R)(\operatorname{det} R)^{\nu} \\
& \left(\int_{S O(n)} t_{i j}^{\omega}\left(O^{-1} K^{-1}\right) \exp \left(2 \pi i \operatorname{tr}\left({ }^{t} \overline{1} O \overline{1} R^{1 / 2} P^{1 / 2}\right)\right) d O\right) d R \\
= & c_{n, m} \sum_{i=1}^{r_{\omega}} \sum_{j, l=1}^{d_{\omega}^{\omega}} t_{j j}^{\omega}\left(K^{-1}\right) \int_{P_{m}} f_{i j}(R) \\
& \left(\int_{S O(n)} t_{i l}^{\omega}\left(O^{-1}\right) \exp \left(2 \pi i \operatorname{tr}\left({ }^{t} \overline{1} O \overline{1} R^{1 / 2} P^{1 / 2}\right)\right) d O\right)(\operatorname{det} R)^{\prime} d R .
\end{aligned}
$$

But $\mathscr{F}$ preserves the space ${ }^{*} \mathcal{K}_{\omega}^{n, m}$. Thus the expression in parentheses above must be zero for $l>r_{\omega}$ and the proof is complete.

Definition 3.3. The Hankel transform of order $\omega$ on $P_{m}$ is the integral transform defined by (3.8). We denote it by $U_{\omega}^{n, m}$. Its kernel $S_{\omega}^{n, m}(R, P)$ is the Bessel kernel of order $\omega$ on $P_{n}$.

Proposition 3.1 establishes a correspondence between ${ }^{*} \mathcal{J}_{\omega}^{n, m}$ and the space $L_{n, m}^{2}(\nu)^{*}$ consisting of $r_{\omega} \times d_{\omega}$ matrix-valued functions $f=\left(f_{i j}\right)$ with $f_{i j} \in L_{n, m}^{2}(\nu)$. An obvious Hilbert space norm in $L_{n, m}^{2}(\nu)^{*}$ is

$$
\|f\|^{2}=\sum_{i, j}\left\|f_{i j}\right\|_{L_{i, m}(v)}
$$

but since $\left(t_{i j}^{\omega}(O)\right)$ is not necessarily unitary $U_{\omega}^{n, m}$ may not be unitary with respect to this norm. On the other hand the compactness of $S O(n)$ implies that $U_{\omega}^{n, m}$ is unitary for some equivalent norm on $L_{n, m}^{2}(\nu)^{*}$. Thus we may write

$$
\mathscr{F}=\sum_{\omega \text { class } m} \bigoplus U_{\omega}^{n, m}
$$


Corollary 3.4. Suppose $\beta$ is a surface Stiefel harmonic of order $\omega$ such that $r_{\omega}$ equals 1. If $F$ in $L^{2}\left(M_{n, m}\right)$ is of the form $f(R) \beta(V)$ then $\hat{F}(X)=g(R) \beta(V)$ and the transform $f(R) \rightarrow g(R)$ is independent of $\beta$ and a choice of basis.

Proof. Since $r_{\omega}=1$, translates of $t_{11}^{\omega}\left(O^{-1}\right)$ generate all of $H_{\omega}^{n, m}$. In particular $\beta(O 1)=\sum_{i} a_{i} t_{11}^{\omega}\left(\left(K_{i}^{-1} O\right)^{-1}\right)$ where $K_{i} \in S O(n)$; thus

$$
f(R) \beta(V)=\sum a_{i} f(R) t_{11}^{\omega}\left(\left(K_{i}^{-1} O\right)^{-1}\right) .
$$

Since $\mathscr{F}$ commutes with rotations, it follows from Theorem 3.2 that $\hat{F}(Y)$ $=g(P) \beta\left(V^{\prime}\right)$ where

$$
g(P)=\int_{R_{m}}\left(\int_{S O(n)} t_{11}^{\omega}\left(O^{-1}\right) \exp \left(2 \pi i \operatorname{tr}\left({ }^{t} \overline{1} O \overline{1} R^{1 / 2} P^{1 / 2}\right)\right) d O\right) f(R)(\operatorname{det} R)^{y} d R .
$$

But $t_{11}^{\omega}\left(O^{-1}\right)$ is determined up to a scalar by the fact that it spans the 1 dimensional space of $S O(n-m)$-invariant functions in $H_{\omega}^{n, m}$. Thus the transform $f \rightarrow g$ is independent of a choice of basis.

In general the explicit form of the Hankel transform $U_{\omega}^{n, m}$ will depend on a choice of basis.

Fixing $n \geq 2 m$ we may combine Corollary 3.4 with Theorem 2.14 to obtain a group theoretic proof of

Theorem 3.5 (Bochner-Herz). Suppose $G$ in $L^{2}\left(M_{n, m}\right)$ is of the form $F(X) p(X)$ where $F(X)$ is radial and $p(X)$ is an $H$-polynomial. Then $\hat{G}(X)=\tilde{F}(X) p(X)$ where $\tilde{F}$ is again radial.

Remark. The case of square matrix space lies outside the multiplicity 1 theory just described. Indeed for $n=m$ we have $r_{\omega}>1$ unless $\omega$ is trivial. Still we might try to recover an analogue of Corollary 3.4 by decomposing $L^{2}\left(M_{n, n}\right)$ further under the action of $O(n) \times O(n)$ on $M_{n, n}=O(n) \times P_{n}$ given by $X \rightarrow a^{-1} X b$, $(a, b) \in O(n) \times O(n)$ (cf. Stein [12, Problem 7]). Since $O(n)$ with this two-sided action is a symmetric space, one expects to confront some kind of multiplicity 1 theory. Nevertheless no analogue of Corollary 3.4 materializes for the following reason.

Since each $R \in P_{m}$ is orthogonally diagonalizable we can write $X=V k^{-1} A k$ where $V$ and $k$ belong to $O(n)$ and

$$
A=\left(\begin{array}{ccc}
\lambda_{1} & & 0 \\
& \ddots & \\
0 & & \lambda_{m}
\end{array}\right)
$$

is a uniquely determined element of the manifold $D_{n}$ of positive definite diagonal matrices in $M_{n, n}$. The action of $G$ on $M_{n, n}$ is such that the $D_{n}$ part is untouched. More precisely, if $X=V k^{-1} A k$ and $g=(a, b) \in O(n) \times O(n)$, then $g X$ $=\left(a^{-1} V b\right)(k b)^{-1} A(k b)$. Since $L^{2}\left(M_{n, n}\right)=L^{2}\left(D_{n}\right) \otimes L^{2}(G)$, where $G=O(n)$ $\times O(n)$, decomposing $L^{2}\left(M_{n, n}\right)$ into subspaces invariant under $X \rightarrow a^{-1} X b$ is equiva- 
lent to decomposing $L^{2}(G)$ under the action $\left(g_{1}, g_{2}\right) \rightarrow\left(a^{-1} g_{1} b, g_{2} b\right)$. This last action is equivalent to the regular representation of $G$. Thus we are still quite far from a multiplicity one theory.

Concluding remark. It has already been suggested that the decompositions (3.3) and (3.9) are not, in general, irreducible. Indeed, the space ${ }^{*} \mathcal{J}_{\omega}^{n, m}$ can be further reduced by allowing $S O(m)$ to act by right multiplication on $M_{n, m}$. Since the Fourier operator commutes with this action the resulting subspaces of ${ }^{*} \mathcal{J}_{\omega}^{n, m}$ are again preserved by $\mathscr{F}$.

4. Explicit Bessel function identities. In the last section we introduced a Hankel transform $U_{\omega}^{n, m}$ for each class $m$ representation of $S O(n)$ by decomposing $L^{2}\left(M_{n, m}\right)$ under the simultaneous action of $\mathscr{F}$ and $S O(n)$. We ask now whether certain of these operators coincide as $n$ and $\omega$ vary? In the classical case $(m=1)$ the answer to this question is given by the simple relation

$$
U_{k}^{n, 1}=i^{k} U_{0}^{n+2 k, 1} .
$$

This identity is due to Bochner and is discussed in [11, Chapter 3, \$3].

We shall generalize (4.1) to $m \geq 1$ by applying the machinery developed in $\$ 2$. Thus we shall once again need to assume $n \geq 2 m$. We shall also employ some straightforward variants of the Laplace transform arguments found in [7]. In particular we shall express our Bessel functions $S_{\omega}^{n, m}$ as inverse Laplace transforms on $P_{m}$ (Theorem 4.2).

We start with a generalization of Theorem $1.3^{\prime}$ in [7].

Proposition 4.1. Suppose

$$
p(X)=\left(\begin{array}{c}
p_{1}(X) \\
\vdots \\
p_{r_{0}}(X)
\end{array}\right)
$$

with $p_{i} \in P^{\omega}$. (Recall that $P^{\omega}$ is the space of vector-valued solid harmonics of order $\omega$ on $M_{n, m}$ introduced in Definition 2.9.) Then

$$
\begin{aligned}
\int_{M_{n, m}} \exp \left(-\pi \operatorname{tr}\left(X Z X^{t}\right)\right) p(X) \exp \left(2 \pi i \operatorname{tr}\left(X Y^{t}\right)\right) d X \\
\quad=(\operatorname{det} z)^{-n / 2} p(i Y) \rho_{\omega}(Z)^{-1} \exp \left(-\pi \operatorname{tr}\left(Y Z^{-1} Y^{t}\right)\right)
\end{aligned}
$$

for all complex symmetric $m \times m$ matrices $Z$ with $\operatorname{Re} Z=A>0$.

Proof. By analytic continuation it suffices to prove the result for $Z=A>0$. So fix $Z=A$. Since $m_{m} \geq 0$ the representation $\rho_{\omega}$ extends from $G L(m, \mathbf{C})$ to $M(m, \mathrm{C})$. Since $p(X A)=p(X) \rho_{\omega}(A)$ for all matrices $A \in M_{m, m}$ the result for $Z=A$ can be made to follow from the special case $Z=I$ by the change of variables $X \rightarrow X A^{1 / 2}$ and $Y \rightarrow Y A^{-1 / 2}$ and the formula $d(X A)=(\operatorname{det} A)^{n} d X$.

We claim now that (4.2) is classical when $Z=I$. Indeed the identity involved is 


$$
\int_{M_{n, m}} e^{-\pi \operatorname{tr}\left(X X^{\prime}\right)} p(X) e^{2 \pi i \operatorname{tr}\left(X Y^{\prime}\right)} d X=e^{-\pi \operatorname{tr}\left(Y Y^{\prime}\right)} p(i Y) .
$$

Since each matrix coefficient of $p(X)$ is harmonic on $M_{n, m}$ and homogeneous of order $|\omega|=\sum_{i=1}^{m} m_{i}$ in the variables $x_{11}, \ldots, x_{n m}$, (4.3) reduces to a collection of classical identities due to Hecke (cf. Theorem 4, p. 71 in [11]). Thus the proof is complete.

Theorem 4.2. Recall $\nu=(n-(m+1)) / 2$. Then

$$
\begin{aligned}
& S_{\omega}^{n, m}(R, P) \rho_{\omega}\left(R^{1 / 2}\right)\left(\operatorname{det} R^{\nu}\right) \\
& =\frac{\pi^{m n / 2} \rho_{\omega}\left(i P^{1 / 2}\right)}{(2 \pi i)^{m(m+1) / 2}} \int_{Z=A_{0}+i B, A_{0}>0}(\operatorname{det} Z)^{-n / 2} \rho_{\omega}\left(\pi^{-1} Z\right)^{-1} \\
& \cdot \exp \left(\operatorname{tr}(R Z)-\operatorname{tr}\left(\pi^{2} P Z^{-l}\right)\right) d Z .
\end{aligned}
$$

Proof. By criteria in [7] the integrals in (4.4) converges for $m_{m}>0$. Applying the integration formulas (3.1) and (3.2) to the identity (4.2) with $p(X)$ as in Theorem 2.13, $X=O \overline{1} R^{1 / 2}$, and $Y=\overline{1} P^{1 / 2}$, we obtain

$$
\begin{gathered}
\int_{R_{m}} e^{-\pi \operatorname{tr}(R Z)}\left(c_{n, m} \int_{\left.s O_{n}\right)} \omega^{*}\left(O^{-1}\right)^{t} \exp \left(2 \pi i \operatorname{tr}\left({ }^{t} \overline{1} O \overline{1} R^{1 / 2} P^{1 / 2}\right)\right) d O\right) \rho_{\omega}\left(R^{1 / 2}\right) \\
\cdot(\operatorname{det} R)^{y} d R \\
=(\operatorname{det} Z)^{-n / 2} \rho_{\omega}\left(i P^{1 / 2}\right) \rho_{\omega}(Z)^{-1} e^{-\pi \operatorname{tr}\left(P Z^{-1}\right)}
\end{gathered}
$$

or

$$
\begin{aligned}
& \int_{P_{m}} e^{-\operatorname{tr}(R Z)} S_{\omega}^{n, m}(R, P) \rho_{\omega}\left(R^{1 / 2}\right)(\operatorname{det} R)^{y} d R \\
& \quad=\pi^{m n / 2}(\operatorname{det} Z)^{-n / 2} \rho_{\omega}\left(i P^{1 / 2}\right) \rho_{\omega}\left(\pi^{-1} Z\right)^{-1} e^{-\pi^{2} t r\left(P Z^{-1}\right)}
\end{aligned}
$$

for all $Z$ with $\operatorname{Re} Z>0$.

Now (4.5) says that for each fixed $P$ the Laplace transform of the (ij)th entry of the matrix $S_{\omega}^{n, m}(R, P) \rho_{\omega}(R)^{1 / 2}(\operatorname{det} R)^{\prime}$ is the $(i j)$ th entry of the matrix

$$
\pi^{m n / 2}(\operatorname{det} Z)^{-n / 2} \rho_{\omega}\left(\pi P^{1 / 2}\right) \rho_{\omega}\left(\pi^{-1} Z\right)^{-1} \exp \left(-\pi^{2} \operatorname{tr}\left(R Z^{-1}\right)\right) \text {. }
$$

(For a discussion of Laplace transforms on $P_{m}$ see $[7,81]$.) Thus by the Cauchy or Laplace inversion formula,

$$
\begin{aligned}
S_{\omega}^{n, m}(R, P) \rho_{\omega}(R)^{1 / 2}(\operatorname{det} R)^{\nu} \\
=\frac{\pi^{m n / 2} \rho_{\omega}\left(i P^{1 / 2}\right)}{(2 \pi i)^{m(m+1) / 2}} \int_{Z-\Lambda_{0}+i B}(\operatorname{det} Z)^{-n / 2} \rho_{\omega}\left(\pi^{-1} Z\right)^{-1} \\
\quad \cdot \exp \left(\operatorname{tr}(R Z)-\operatorname{tr}\left(\pi^{2} P Z^{-1}\right)\right) d Z .
\end{aligned}
$$

This completes the proof. 
Now recall the correspondence which assigns, to functions in ${ }^{*} C_{\omega}^{n, m}, r_{\omega} \times d_{\omega}$ matrices $\left(f_{i j}\right)$ and let ${ }^{0}{ }^{0} C_{\omega}^{n, m}$ denote the subspace of ${ }^{*}{ }^{*} C_{\omega}^{n, m}$ corresponding to the (first) column space $\left(f_{i 1}\right)={ }^{0} L_{n, m}(v)$. The following theorem describes when the Hankel transforms $U_{\omega}^{n, m}$ and $U_{\omega^{\prime}}^{n^{\prime}, m}$ coincide on the spaces ${ }^{0 \int_{C_{\omega}}^{n, m}}$ and ${ }^{0} \mathcal{S C}_{\omega^{n}, m} n^{\prime}$.

Theorem 4.3 (Generalized Bochner relations). If $(n, \omega)$ denotes the pair ( $n$, $\left.\left(m_{1}, \ldots, m_{m}\right)\right)$ and $\left(n^{\prime}, \omega^{\prime}\right)$ the pair $\left(n+2 m_{m}, \omega-m_{m}\right)$ (where $\omega-m_{m}=\left(m_{1}\right.$ $\left.\left.-m_{m}, m_{2}-m_{m}, \ldots, 0\right)\right)$ then

$$
U_{\omega}^{n, m}=c_{\omega}^{m} U_{\omega^{\prime}}^{n^{\prime}, m}
$$

where $c_{\omega}^{m}=i^{m m_{m}}$.

Proof. The operators $U_{\omega}^{n, m}$ and $U_{\omega^{\prime}}^{n^{\prime}, m}$ are defined on ${ }^{0} L_{n, m}^{2}(\nu)$ and ${ }^{0} L_{n^{\prime}, m}^{2}\left(\nu^{\prime}\right)$ respectively by formula (3.6). We claim that the isometry

$$
A: f(R) \rightarrow \sqrt{c_{n, m} / c_{n^{\prime}, m}} f(R)(\operatorname{det} R)^{-m_{m / 2}}
$$

which maps ${ }^{0} L_{n, m}^{2}(\nu)$ onto ${ }^{0} L_{n+2 m_{m}, m}^{2}\left(\nu+m_{m}\right)$ realizes the equivalence between $U_{\omega}^{n, m}$ and $U_{\omega^{\prime}}^{n^{\prime}, m}$. Indeed $\rho_{\omega^{\prime}}(Z)=(\operatorname{det} Z)^{-m_{m}} \rho_{\omega}(Z)$ since $\rho_{\left(m_{m}, \ldots, m_{m}\right)}(Z)=(\operatorname{det} Z)^{m_{m}}$. Thus by a straightforward application of Theorem 4.2,

$$
\begin{gathered}
\left(A^{-1} U_{\omega^{\prime}}^{n^{\prime}, m} A f\right)(P)=(\operatorname{det} P)^{m_{m^{\prime}} / 2} \int_{P_{m}} S_{\omega^{\prime}}^{n^{\prime}, m}(R, P) f(R)(\operatorname{det} R)^{\gamma}(\operatorname{det} R)^{-m_{m} / 2} d R \\
=(\operatorname{det} P)^{m_{m} / 2} \int_{\mathbb{R}_{m}}\left(\frac{\pi^{m n^{\prime} / 2} \rho_{\omega^{\prime}}\left(i P^{1 / 2}\right)}{(2 \pi i)^{m(m+1) / 2} \int(\operatorname{det} Z)^{-n / 2} \rho_{\omega^{\prime}}\left(\pi^{-1} Z\right)^{-1}}\right. \\
\left.\cdot \exp \left(\operatorname{tr}(R Z)-\operatorname{tr}\left(\pi^{2} P Z^{-1}\right) d Z\right) \rho_{\omega^{\prime}}\left(R^{1 / 2}\right)^{-1}\right) \\
\cdot f(R)(\operatorname{det} R)^{-m_{m} / 2} d R \\
=i^{-m m_{m}} \int_{P_{m}}\left(\frac{\pi^{m n / 2} \rho_{\omega}(i P 1 / 2)}{(2 \pi i)^{m(m+1) / 2} \int(\operatorname{det} Z)^{-n / 2} \rho_{\omega}\left(\pi^{-1} Z\right)^{-1}}\right. \\
\left.\cdot \exp \left(\operatorname{tr}(R Z)-\operatorname{tr}\left(\pi^{2} P Z^{-1}\right) d Z\right) \rho_{\omega}\left(R^{1 / 2}\right)^{-1}\right) \\
\cdot f(R) d R \\
=i^{-m m_{m}} \int S_{\omega}^{n, m}(R, P) f(R)(\operatorname{det} R)^{b} d R=i^{-m m_{m}} U_{\omega}^{n, m} .
\end{gathered}
$$

This completes the proof.

When $m=1$ and $\omega=(k, 0, \ldots, 0), C_{\omega}^{m}=i^{k}$. Thus Theorem 4.4 reduces to Bochner's identity (4.1) when $m=1$. 


\section{REFERENCES}

1. S. Bochner, Theta relations with spherical harmonics, Proc. Nat. Acad. Sci. U.S.A. 37 (1951), 804-808. MR 14, 43.

2. H. Boerner, Representations of groups, North-Holland, Amsterdam, 1963.

3. E. Cartan, Sur la determination d'un système orthogonal complet dans un espace de Riemann symétrique clos, Rend. Circ. Mat. Palermo 53 (1929), 217-252.

4. S. Gelbart, Harmonics on Stiefel manifolds and generalized Hankel transforms, Bull. Amer. Math. Soc. 78 (1972), $451-455$.

5. - Holomorphic discrete series for the real symplectic group, Invent. Math. 19 (1973), 49-58.

6. S. Helgason, Invariants and fundamental funtions, Acta Math. 109 (1963), 241-258. MR 29 \#3581.

7. C. Herz, Bessel functions of matrix argument, Ann. of Math. (2) 61 (1955), 474-523. MR 16, 1107.

8. B. Kostant, Lie group representations on polynomial rings, Amer. J. Math. 85 (1963), 327-404. MR 28 \# 1252.

9. D. Levine, Systems of singular integrals on spheres, Trans. Amer. Math. Soc. 144 (1969), 493-522.

10. H. Maass, Spherical functions and quadratic forms, J. Indian Math. Soc. 20 (1956), 117-162. MR $19,252$.

11. E. Stein, Singular integrals and differentiability properties of functions, Princeton Math. Series, no. 30, Princeton Univ. Press, Princeton, N.J., 1970. MR 44 \# 7280.

12. - Some problems in harmonic analysis suggested by symmetric spaces and semi-simple groups,

Prof. Internat. Congress Math. (Nice, 1970), vol. 1, Gauthier-Villars, Paris, 1971, pp. 173-190.

13. R. Strichartz, The Fourier decomposition of $L^{2}(S O(n) / S O(n-m))$ (to appear).

14. A. Weil, Sur certains groupes d’opérateurs unitaires, Acta Math. 111 (1964), 143-211. MR 29 \#2324.

15. H. Weyl, The classical groups. Their invariants and representations, 2 nd ed., Princeton Univ. Press, Princeton, N.J., 1946.

School of Mathematics, Institute for Advanced Study, Princeton, New Jersey 08540

Current Address: Department Of Mathematics, Cornell University, Ithaca, New York 14850 